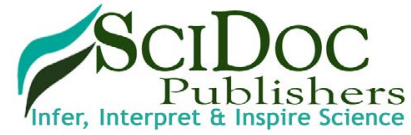

International Journal of Anesthesiology \& Research (IJAR)

ISSN 2332-2780

\title{
Acute Hypotensive Transfusion Reaction in a Patient Taking Angiotensin Converting Enzyme Inhibitors Therapy: A Case Report and Literature Review
}

Dalia $\mathrm{A}^{1 *}$, Alicia A. Gonzalez Z1 , Ostrum $\mathrm{SG}^{2}$, Stoicea $\mathrm{N}^{1}$, Yehsakul $\mathrm{D}^{1}$

${ }^{1}$ The Ohio State University Wexner Medical Center, Department of Anesthesiology, Columbus OH, USA.

${ }^{2}$ The Ohio State University Wexner Medical Center, College of Medicine, Columbus OH, USA.

\section{Abstract}

Acute Hypotensive Transfusion Reaction (AHTR) is characterized by the sudden onset and isolated drop in blood pressure which resolves a few minutes after discontinuing the transfusion without additional therapy. The pathogenesis of this reaction is generated by an increase in Bradykinin (BK), a state which may be encouraged by use of an Angiotensin Converting Enzyme Inhibitor (ACEi). We report a case of acute and severe hypotension in a patient on ACEi therapy that we hypothesized to be an AHTR based upon the following evidence: The symptomatology presentation at the time of the transfusion, the negative laboratory workup for other common culprits, and the tolerance of subsequent transfusions after stopping the ACEi. While the discontinuation of an ACEi when thought to be contributory to an AHTR has been recommended, to the best of our knowledge, we describe the first reported case of a likely AHTR evident where this was implemented and successful in a surgical setting. Herein we detail our patient's possible AHTR event and following medical course, the rational for our conclusion and clinical actions, and a review of the recent AHTR literature.

Keywords: Acute Hypotension; Angiotensin Converting Enzyme; Bradykinin; Hypotension; Transfusion.

\section{Introduction}

Acute hypotension is one of the most common and striking manifestation of transfusion reactions caused by contamination of blood products, acute hemolysis, transfusion-associated lung injury, and anaphylaxis [1]. Acute Hypotension Transfusion Reaction (AHTR) is characterized by the sole symptom of severe hypotension following a transfusion, and the resolution of such hypotension subsequent to the discontinuation of the transfusion. Although the case later described involved Packed Red Blood Cells (pRBCs) and Fresh Frozen Plasma (FFP), AHTR was first described in the 1970's with platelet derived products, and became even more prevalent in the early 90's with the introduction of bedside leukoreduction filters [1-3]. Though the hypotension of AHTR is often described as an entirely isolated reaction other symptoms such as lightheadedness, dizziness, and anxiety associated with the drop in blood pressure may also occur [4].

The literature provides insight into the key players involved in the AHTR reaction, though the pathophysiology of AHTR is not fully understood. The hypothesis centers around the increased levels of the vasoactive peptide, Bradykinin (BK), that occurs in a transfusion recipient after the blood or blood product makes contact with a negatively charged leukoreduction filter $[2,5-7]$. Other items that may act as a negatively charged surface include: dialysis membranes, dextran sulfate, glass, kaolin, and blood filters $[1-3,8]$. When blood or blood products come into contact with these negatively charged materials a cascade is initiated known as the Kallikrein-Kinin system. Initially Factor XII (Hageman Factor) is activated once in contact with the negatively charged surface, becoming known as activated Factor XII (XIIa). XIIa cleaves pre-Kallikrein forming Kallikrein [8]. At this point in the reaction, Kallikrein cleaves High-Molecular-Weight Kininogen (HMW-Kininogen) releasing BK. BK exerts its hypotensive effects through the BK-2 receptors intravascularly causing systemic hypotension via profound vasodilitation, especially in patients taking Angiotensin Converting Enzyme Inhibitors (ACEi) therapy [1,9] (Figure 1).

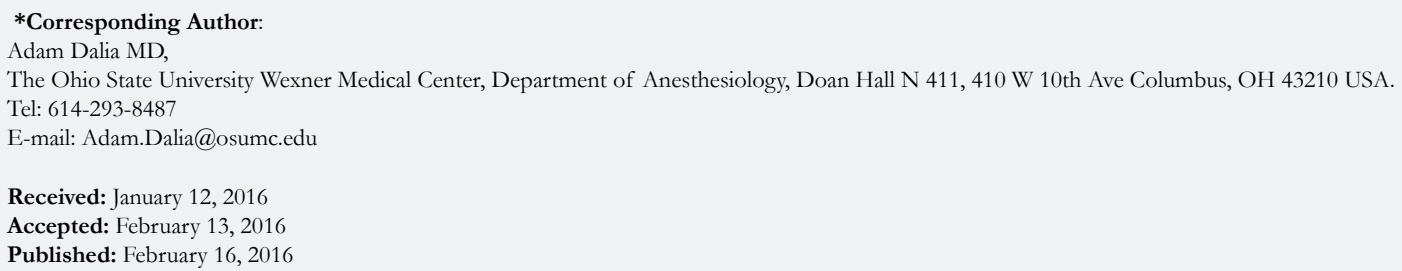

Copyright: Dalia $\mathbf{A}^{\circ}$ 2016. This is an open-access article distributed under the terms of the Creative Commons Attribution License, which permits unrestricted use, distribution and reproduction in any medium, provided the original author and source are credited. 
Figure 1. Bradykinin Metabolism Pathway.

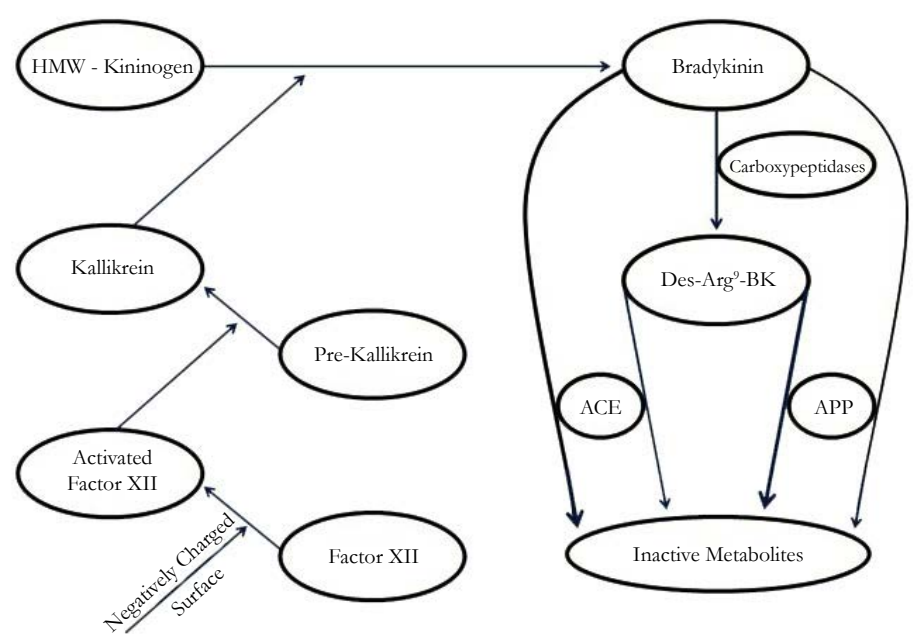

Factor XII, once in contact with a negatively charged surface, becomes activated Factor XII. Factor XIIa furthermore activates the bound pre-Kallikrein forming Kallikrein. Kallikrein cleaves High-Molecular-Weight Kininogen (HMW-Kininogen), which in turn releases Bradykinin (BK) from HMWK. BK metabolism is accomplished through Carboxypeptidases to form the activate metabolite Des-Arg9-BK. BK is then metabolized by Angiotensin Converting Enzyme (ACE) and Aminopeptidase P (APP). Furthermore, Des-Arg9-BK [1-9] is metabolized by ACE and APP to form inactive metabolites.

Normally, BK is rapidly metabolized by Angiotensin Converting Enzyme (ACE), aminopeptidase P (APP) or carboxypeptidases. ACE is the primary metabolizer of $\mathrm{BK}$ accounting for nearly $75 \%$ of its metabolism, the remaining $25 \%$ is done so by APP or carboxypeptidases [1, 4, 5]. Interestingly, carboxypeptidase metabolism yields Des-Arg'-BK, a vasoactive metabolite with an affinity for the BK-1 receptor, which may also contribute to hypotension. Des-Arg ${ }^{9}-\mathrm{BK}$ is inactivated by both ACE and APP [9]. Recently, there is a growing amount of literature suggesting the importance of APP activity in patients with respect to AHTR. The short half-life of BK, approximately 49 seconds, and its active metabolite Des-Arg'-BK, approximately 325 seconds, is responsible for the rapid resolution of AHTR after discontinuation of the transfusion. Notably, the majority of Des-Arg'-BK is metabolized by the APP enzyme with a small contribution by the ACE pathway $[2,10,11]$. This may account for the finding that although APP activity is only responsible for a fraction of $\mathrm{BK}$ metabolism, the genetic competency of the enzyme plays a key role in preventing unabashed BK activity $[1,4]$.

What follows is a case of likely AHTR and its relevance to the current body of knowledge and theory of AHTR.

\section{Case Report}

A 69 y/o Caucasian female presented for a lumbar laminectomy with fusion and L2 vertebrectomy for symptomatic spinal stenosis secondary to a renal cell carcinoma metastatic lesion. Her past medical history was notable for hypertension, hypothyroidism, gastroesophageal reflux, and diabetes mellitus type II. Home medications included insulin, omeprazole, and lisinopril.

An arterial line (A-line), large bore multi-lumen central venous catheter (CVC), and a rapid transfuser were utilized in anticipation of massive blood loss. The patient had a baseline anemia and demonstrated notable pulse pressure variation on A-line and as a result transfusion with FFP and pRBCs in citrate-phosphatedextrose-adenine (CPDA) in a 1:1 ratio was initiated via rapid transfuser set after the induction of general anesthesia.

At this moment, the patient quickly became hypotensive (Mean Arterial Pressure - MAP fell to below 30mmHg, MAP $95 \mathrm{mmHg}$ immediately prior). The transfusion was halted and $1 \mathrm{mg}$ IV epinephrine was administered to the patient. Perfusion pressure recovered and surgery continued. Hydrocortisone, diphenhydramine, and famotidine were administered IV. Blood products and patient blood and urine samples were sent for immediate evaluation. Laboratory did not reveal blood mismatch or any suggestion of hemolytic reaction (Figure 2).

Transfusion was again attempted with new blood products and rapid transfuser set. With the upmost vigilance blood pressure was monitored. Hypotension again resulted (MAP of $80 \mathrm{mmHg}$ declining to $44 \mathrm{mmHg}$ ), but responded to small doses of IV epinephrine (approximately $20 \mathrm{mcg}$ at a time). However, significant tachycardia resulted. Transfusion was soon stopped and hemodynamics normalized. Again laboratory samples were drawn and were unrevealing.

Because no safe means of blood transfusion could be established, the surgical procedure was truncated to a laminectomy and fusion. Postoperatively the patient became symptomatically anemic, exhibiting hypotension and tachycardia. Hemoglobin nadir was $6.1 \mathrm{~g} / \mathrm{dL}$. Consultation with pathology and blood bank suggested an AHTR especially in light of the recent use of lisinopril (morning dose received day of surgery). Further administration of ACEi was held and patient advised against their use. The patient tolerated washed pRBC's without hemodynamic compromise following additional prophylactic administration of IV hydrocortisone, diphenhydramine, and famotidine. The patient was later discharged and completion of the vertebrectomy was planned for a subsequent admission.

Discussion with pathology suggested that AHTR was probable likely secondary to lisinopril. Pathology also entertained the possibility of a very rare reaction to adenine preservative. 
Figure 2. HR and MAP Monitoring.

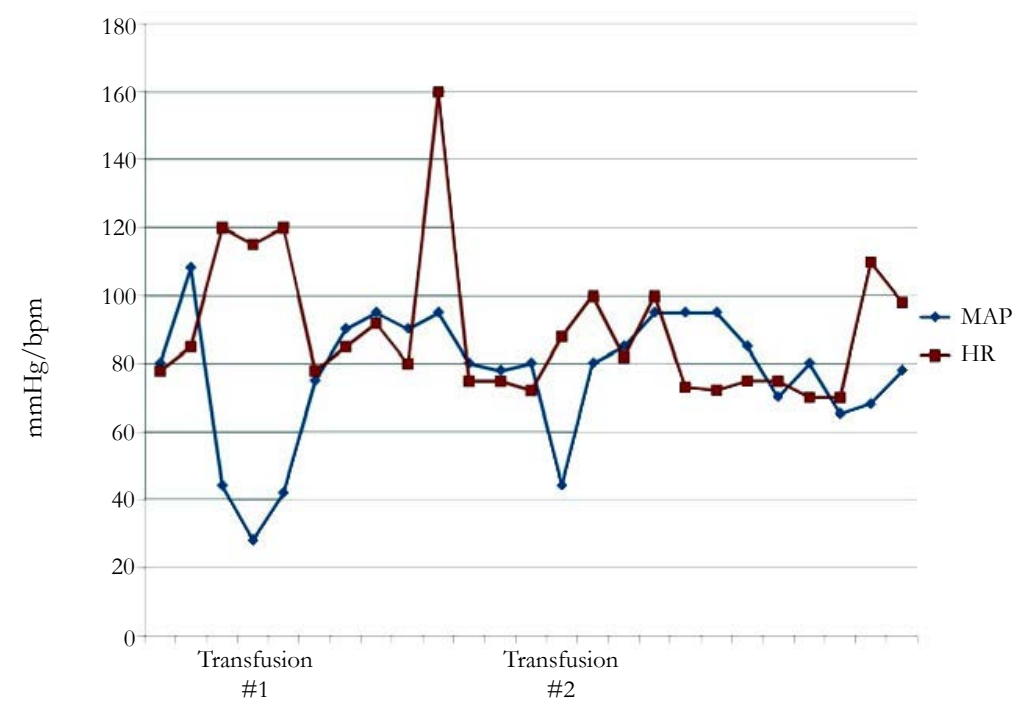

Mean arterial pressure (MAP) and heart rate (HR) monitoring every 15 minutes before and after \#1 and \#2 transfusion of 2units of Packed Red Blood Cells and 2 units of Fresh Frozen Plasma through Belmont Rapid Transfuser causing AHTR episodes and treated with IV Epinephrine respectively.

The patient was brought back for a trial transfusion of blood. Pathology though it most prudent to utilize CPD-pRBC for this trial. In the awake patient, a unit of CPD-pRBC was administered without consequence. After further discussion with pathology, they agreed that AHTR was still the most likely culprit and that adenine free blood was not likely to be required. As lisinopril had been discontinued for several weeks, the patient theoretically would tolerate standard blood products; the patient consented to trial CPDA blood.

The patient was readmitted for surgery, this time for L2 vertebrectomy alone. Several CPD-pRBCs were made available. Following general anesthesia, a CVC was placed and the patient was administered CPDA-pRBC with FFP in a 1:1 ratio via the same rapid transfuser. No prophylaxis was administered. Slight relative hypotension was noted (MAP declined from $120 \mathrm{mmHg}$ to $100 \mathrm{mmHg}$ ), but was physiologically unconcerning and not prohibitive. The vertebrectomy was successfully accomplished with the administration of 13 units of CPDA-pRBCs. Patient remained hemodynamically stable throughout the surgery, and was immediately extubated. Surgical recovery was uneventful.

\section{Informed Consent}

Verbal informed consent was obtained from the patient described in this case report per our local Institutional Review Board Guidelines.

\section{Discussion}

Current protocols for transfusion products require the storage of negatively-reduced blood products between filtration and transfusion in order to avoid the occurrence of AHTRs [12]. During this storage period, $\mathrm{BK}$ generated in the reduction process undergoes spontaneous degradation. Recent literature has also described other ATHR episodes accompanying prestorage leukoreduced blood products [12-15]. In our case, the patient received prestorage leukoreduced product, thought to be deficient in $\mathrm{BK}$.
In 2004, AHTRs were reported with prestorage leukoreduced whole blood products in two patients undergoing radical prostatectomy [5]. More recently, investigators reviewing US hemovigilance data from 2011 to 2012 on reports of hypotensive transfusion reactions identified 35 cases of AHTR even in the setting of prestorage leukoreduced blood products [12]. Thus, those patients who exhibited hypotension with transfusion during extracorporeal membrane oxygenation (ECMO) or cardiopulmonary bypass (CPB) fit best within our current understanding of AHTR. CPB and ACEi have long been associated with AHTR and are thought to each cause dysfunction in BK metabolism $[12,16]$. With these extracorporeal systems, blood flow is directed away from the pulmonary circulation avoiding ACE secretion in the lungs, and thus circumventing BK degradation [12]. Dialysis, another extracorporeal circuit, has also been well illustrated to increase BK levels through the incorporation of negatively-charged membranes [2, 12].

The continuation of AHTR even with prestorage leukoreduced blood products draws into question the accepted pathophysiology and preventive measures for AHTR. Furthermore, the patient was positive for metastatic renal carcinoma, in the absence of cardiopulmonary dysfunction. AHTR has been described in cancer patients $[12,13]$, but the relationship between cancer and AHTR remains poorly understood.

Findings since the original implication of the KallikreinKinin system continue to alter our understanding of AHTR pathophysiology. Genetic polymorphisms that significantly impact BK and Des-Arg ${ }^{9}$-BK metabolism have been described. In a subset of patients undergoing CABG without ACEi treatment, AHTRs were associated with a single nucleotide polymorphism, C-2399A, in the APP gene. This C-2399A polymorphism was linked to decreased APP activity [12]. Patients undergoing liver transplant and on ACEi treatment with AHTRs were also found to exhibit genetic variances resulting in altered APP activity [9, 171 . 
Furthermore, Crews et al., postulated that recurrent blood transfusions of leukoreduced blood products could result in the upregulation of $\mathrm{B} 1$ receptors, thus increasing the sensitivity of the patient to BK and Des-Arg'-BK [4]. This finding would imply that susceptibility to AHTR recurrence could be dynamic in the patients themselves.

The sole management needed for an AHTR is discontinuation of the offending transfusion to prevent further intravascular release of BK $[1,17,18]$. AHTR can be observed as early as 30 seconds into the transfusion but can also take as long as 10 minutes, the mean time described in the literature is 4 minutes with a mean drop in systolic blood pressure to $53 \mathrm{mmHg}[2,16]$. In most cases no further treatment is needed. If the hypotension is severe and sustained, supportive therapy may be helpful. Vasoactive drugs, like phenylephrine or vasopressin, can improve the hemodynamic profile during these brief hypotensive episodes $[1,12,17]$.

The patient's initial hypotensive reaction to intra-operative blood products yielded a wide differential. First and foremost a transfusion mismatch was considered. This possibility was eliminated with confirmatory retesting of the patient's blood type, lack of evidence for hemolysis from serum and urine samples, and a negative direct antigen test. An anaphylactic or anaphylactoid reaction was unlikely. Aside from hypotension, the patient exhibited no other corresponding signs or symptoms such as rash or bronchospasm. IgA deficiency was considered, but also deemed unlikely. No anti-IgA was detected and postoperative IgA levels were within normal limits. Lastly, bacterial contamination was considered. This too was not very likely. The patient exhibited hypotension twice with two different sets of blood products and two different sets of transfusion lines. The patient also did not demonstrate any fever. Finally, the patient tolerance of CPDA-pRBCs during her second operation once her ACEi was held helps to support our theory of AHTR.

Indeed, the case we present is not definitively an example of AHTR. AHTR is generally a diagnosis of exclusion. Directly proving hypotension is secondary to increased circulating $\mathrm{BK}$ generated via blood transfusion is not commonly feasible. But we believe as a whole, our theory holds up to scrutiny. Nonetheless, there are points of action described in the case that necessitate explanation.

First, we treated our patient's hypotensive events with epinephrine. And secondly, we encouraged the patient to trial CPDA-pRBCs with her completion vertebrectomy. The literature notes that AHTR resolves with the cessation of blood transfusion and that it should not be treated as anaphylactic reaction. However, at that time an accurate diagnosis could not be established. In that instance, life threatening hemolytic or anaphylactic reactions must be considered and treatment accordingly is prudent. In addition, marked hypotension in a fully anesthetized patient adds the complexity of being unable to assess the patient for neurologic injury from hypoperfusion. Lastly, our patient was to have a renal carcinoma metastasis removed with her vertebrectomy, an endeavor that invites the risk of substantial blood loss. Washed pRBCs have been described as a potentially safe alternative to more commonly prepared pRBCs [4] and following premedication our patient did demonstrate tolerance to this therapy. However, washed $\mathrm{pRBCs}$ take several hours to prepare and have a short shelf life. CPD-pRBCs are more readily available, but are nowhere near as abundant and quickly prepared as CPDA-pRBCs at our institution. As such, proving that our patient could receive CPDApRBCs enhanced the likelihood of success of our undertaking with minimal risk. Safety was ultimately our guide.

The unicity of our case report is assured by the ability of diagnosing a rare pathologic condition, and implementing a treatment strategy explored only theoretically in the medical literature. Although blood products have been administered upon discontinuation of ACEi in the postoperative setting [2, 4], to our knowledge this is the first report when a patient has successfully received blood products in a subsequent surgical intervention, after discontinuation of ACEi treatment. Therefore, by advising the patient to discontinue her ACE inhibitor prior to returning to the operating room, we demonstrated a technique that has not been practiced but only described in the literature as a possible method to avoid future AHTR's.

\section{Conclusion}

The increased use of an ACEi for the treatment of hypertension has brought to light its contributory role in some instances of AHTR. While not all patients taking an ACEi and receiving blood transfusion exhibit hypotension, the rare but notable association has helped to forge a better understanding of AHTR pathophysiology. The initial treatment of AHTR is to halt transfusion and to support the patient's blood pressure if indicated. The use of washed pRBCs may be tolerated. In patients taking an ACEi, suspending the drug or switching to another antihypertensive class may avert future AHTR in susceptible patients.

\section{References}

[1]. Bruno DS, Herman JH (2006) Acute hypotensive transfusion reactions. Lab Med 37(9): 542-545.

[2]. Cyr M, Eastlund T, Blais C, Rouleau JL, Adam A (2001) Bradykinin metabolism and hypotensive transfusion reactions. Transfusion 41(1): 136-150.

[3]. Perrotta PL, Synder EL (2001) Non-infectious complications of transfusion therapy. Blood Rev15(2): 69-83.

[4]. Crews WS Jr, Kay JK, Herman JH (2014) Washed RBCs Prevent Reccurrent Acute Hypotensive Transfusion Reactions. Am J Clin Pathol 141(2): 285-287.

[5]. Arnold DM, Molinaro G, Warkentin TE, DiTamasson J, Webert KE, et al. (2004) Hypotensive transfusion reactions can occur with blood products that are leukoreduced before storage. Transfusion 44(9): 1361-1366.

[6]. Sreelakshmi TR, Eldridge J (2010) Acute hypotension associated with leucocyte depletion filters during cell salvaged blood transfusion. Anaesthesia 65(7): $742-744$.

[7]. Sharma RR, Marwaha N (2010) Leukoreduced blood components: Advantages and strategies for its implementation in developing countries. Asian J Transfus Sci 4(1): 3-8.

[8]. Kaplan AP, Joseph K, Shibayama Y, Nakazawa Y, Ghebrehiwet B, et al. (1998) Bradykinin formation. Plasma and tissue pathways and cellular interactions. Clin Rev Allergy Immunol 16(4): 403-429.

[9]. Doria C, Elia ES, Kang Y, Adam A, Desormeaux A, et al. (2008) Acute Hypotensive Transfusion Reaction During Liver Transplantation in a Patient on Angiotensin Converting Enzyme Inhibitors from Low Aminopeptidase $P$ Activity. Liver Transpl 14(5): 684-687.

[10]. Cyr M, Hume HA, Champagne M, et al. (1999) Anomaly of the des-Arg9bradykinin metabolism associated with severe hypotensive reactions during blood transfusion: a preliminary report. Transfusion 39: 1084-1088.

[11]. Gräfe M, Bossaller C, Graf K, Auch-Schwelk W, Baumgarten CR, et al. (1993) Effect of angiotensin-converting-enzyme inhibition on bradykinin metabolism by vascular endothelial cells. Am J Physiol $264(5$ Pt 2): H14931497

[12]. Pagano MB, Ness PM, Chajewski OS, King KE, Wu Y, et al. (2015) Hypotensive transfusion reactions in the era of prestorage leukoreduction. Trans- 
fusion 55(7): 1668-1674.

[13]. Quillen K (2000) Hypotensive Transfusion Reactions in Patients Taking Angiotensin-Converting-Enzyme Inhibitors. N Engl J Med 343: 1422-1423.

[14]. Fried MR, Eastlund T, Christie B, Mullin GT, Key NS (1996) Hypotensive reactions to white cell-reduced plasma in a patient undergoing angiotensinconverting enzyme inhibitor therapy. Transfusion 36(10): 900-903.

[15]. Hume HA, Popovsky MA, Benson K, Glassman AB, Hines D, et al. (1996) Hypotensive reactions: a previously uncharacterized complication of platelet transfusion? Transfusion 36(10): 904-909.

[16]. Mair B, Leparc GF (1998) Hypotension Reactions Associated with Plate- let Transfusions and Angiotensin-Converting Enzyme Inhibitors. Vox Sang 74(1): 27-30.

[17]. Kalra A, Palaniswamy C, Patel R, Kalra A, Selvaraj DR (2012) Acute Hypotensive Transfusion Reaction With Concomitant Use of Angiotensin-Converting Enzyme Inhibitors: A Case Report and Review of the Literature. Am J Ther 19(2): e90-94.

[18]. Alving BM, Hojima Y, Pisano JJ, Mason BL, Buckingham RE Jr, et al. (1978) Hypotension associated with prekallikrein activator (Hageman-factor fragments) in plasma protein fraction. N Engl J Med 299(2): 66-70. 